Chirurg 2013 $84: 1000$

DOI 10.1007/s00104-013-2631-8

Online publiziert: 13. Oktober 2013

(c) Springer-Verlag Berlin Heidelberg 2013

J. Reibetanz · C.-T. Germer

Klinik für Allgemein-, Viszeral-, Gefäß- und Kinderchirurgie, Universitätsklinik Würzburg

\title{
Neoadjuvante Chemotherapie beim lokal fortgeschrittenen Kolonkarzinom
}

\section{Erste Ergebnisse der FOxTROT-Studie}

\section{Originalpublikation}

FOxTROT Collaborative Group (2012) Feasibility of preoperative chemotherapy for locally advanced, operable colon cancer: the pilot phase of a randomised controlled trial. Lancet Oncol 13:1152-1160

\section{Hintergrund}

Die präoperative (Radio-)Chemotherapie hat sich - verglichen mit der postoperativen Behandlung - beim Ösophagus-, Magen- und Rektumkarzinom u. a. aufgrund besserer Verträglichkeit, höherer Ansprechraten, höherer R0-Resektabilität und exakterer Prognoseabschätzung durch Response-Beurteilung als die effektivere Therapie herausgestellt. Derartige neoadjuvante Therapiekonzepte existieren für das Kolonkarzinom derzeit noch nicht.

\section{Fragestellung der Studie}

Ziel der FOxTROT-Studie (Fluoropyrimidine Oxaliplatin and Targeted Receptor Pre-Operative Therapy) war die Beurteilung der Durchführbarkeit, Sicherheit und Effektivität einer neoadjuvanten 6-wöchigen Kombinationschemotherapie (CTx) beim lokal fortgeschrittenen Kolonkarzinom. Hierzu wurden im Zeitraum 2008 bis 2010 insgesamt 150 Patienten mit T3/4Kolonkarzinomen nach 2:1-Randomisierung entweder perioperativ [präoperativ 3 Zyklen 5-Fluoruracil + Oxaliplatin (5-FU$\mathrm{Ox}) \rightarrow$ Operation $\rightarrow 9$ Zyklen 5-FU-Ox] oder konventionell postoperativ [Operation $\rightarrow 12$ Zyklen 5-FU-Ox] behandelt. KRAS-Wildtyp-Patienten wurden zudem im Verhältnis 1:1 für die Therapie mit $\mathrm{Pa}$ nitumumab randomisiert.

\section{Ergebnisse}

Von 150 eingeschlossenen Patienten wurden 99 Patienten in die perioperative (Intervention) und 51 Patienten in die postoperative Chemotherapiegruppe (Kontrolle) randomisiert, wobei sich beide Gruppen nicht in den Tumorcharakteristika unterschieden. 96\% (95/96) der Patienten der Interventionsgruppe begannen und $89 \%$ (85/95) beendeten die präoperative CTx protokollgemäß, alle 99 Patienten konnten (nach durchschnittlich 61 Tagen ab Beginn der CTx) reseziert werden. Postoperativ führten $83 \%$ (82/99) die CTx fort, 82\% (67/82) hiervon beendeten alle Zyklen. Insgesamt war die Therapie-Compliance der Interventionsgruppe besser als die der Kontrollgruppe (Beginn der CTx: 96 vs. 78\%, p=0,001; Komplettierung aller Zyklen: $68 \%$ vs. $57 \%$, $\mathrm{p}=0,19)$. Verglichen mit der Kontrollgruppe führte die neoadjuvante Therapie zu einem signifikanten Downstaging $(\mathrm{p}=0,04)$, einem selteneren Befall zentraler Lymphknoten ( $1 \%$ vs. $20 \%$, p<0,0001), weniger Fällen mit positivem Resektionsrand ( $4 \%$ vs. $20 \%, p=0,002)$ und einem höheren Grad an Tumorregression (moderat oder größer: $31 \%$ vs. $2 \%$, $\mathrm{p}=0,0001$, inklusive zweier Fälle mit „complete response“). Beide Gruppen unterschieden sich nicht signifikant hinsichtlich der perioperativen Komplikationsrate (14\% vs. $12 \%, \mathrm{p}=0,81$ ).

\section{Diskussion und Fazit des Reviewers}

Erste Ergebnisse der FOxTROT-Studie zeigen, dass eine 6-wöchtige neoadjuvante
CTx beim lokal fortgeschrittenen Kolonkarzinom mit einer exzellenten Therapie-Compliance einhergeht und sich hinsichtlich der Parameter Downstaging, R0-Resektabilität, Tumorregression und Befall des Grenzlymphknotens vorteilhaft auswirkt, ohne Zugeständnisse an die perioperative Komplikationsrate. Onkologische Langzeitergebnisse stehen aus, entsprechende Studien scheinen aber aufgrund der vorgelegten Daten mehr als gerechtfertigt. Darüber hinaus konnte FOxTROT zeigen, dass mittels Mehrzeilencomputertomographie (MSCT) eine adäquate präoperative $\mathrm{Pa}$ tientenselektion für die Neoadjuvanz möglich ist, eine unabdingbare Vorraussetzung, um Übertherapie zu vermeiden. Die „Achillesferse“ des neoadjuvanten Therapieansatzes liegt damit in der Exaktheit des präoperativen radiologischen Stagings, was die MSCT zur obligaten Untersuchung beim Kolonkarzinom machen würde. Abdomensonographie und Thoraxröntgen - wie derzeit noch als Basisuntersuchung von der S3-Leitlinie „Kolorektales Karzinom" empfohlen - wären dann wohl eher als „,historisch“ anzusehen.

\section{Korrespondenzadresse}

Prof. Dr. C.-T. Germer

Klinik für Allgemein-, Viszeral-, Gefäß- und Kinderchirurgie, Universitätsklinik Würzburg, Oberdürrbacher Str. 6, 97080 Würzburg germer_c@klinik.uni-wuerzburg.de

\section{Einhaltung ethischer Richtlinien}

Interessenkonflikt. J. Reibetanz und C.T. Germer geben an, dass kein Interessenkonflikt besteht. 